Preprint n.956

July 15, 1993.
Dipartimento di Fisica Universitá di Roma "la Sapienza"

I.N.F.N. sezione di Roma

\title{
TeV Muons in Hadronic Showers
}

\author{
P. Lipari \\ I.N.F.N., Sezione di Roma, and \\ Dipartimento di Fisica, Università di Roma "la Sapienza", \\ Piazzale A. Moro 2, I-00185 Roma, Italy
}

\begin{abstract}
From the study of the multiple muon events in deep underground detectors, it is possible to extract information about the spectrum and composition of the primary cosmic rays. In this work the number of $\mathrm{TeV}$ muons produced by a primary cosmic ray of a given energy and zenith angle is computed using analytic and montecarlo methods, for a family of simplified models as description of the properties of hadronic interactions. The effects of the uncertainties in our knowledge of the hadronic cross sections in the calculation of $\mathrm{TeV}$ muons are discussed.
\end{abstract}




\section{Introduction}

Deep underground detectors measure events with several nearly parallel muons [1, 2, 3], separated by few meters [4. The muons are produced by the decay of charged pions and kaons in the hadronic showers induced by primary cosmic rays. From the study of these events it is possible to obtain information about the spectrum and composition of cosmic rays in the energy region $\left(10^{14} \lesssim E_{0} \lesssim 10^{17} \mathrm{eV}\right)$.

The shower produced by a nucleus of total energy $E_{0}$ and mass number $A$ can be described with reasonable accuracy as the superposition of $A$ proton showers each of energy $E_{0} / A$. Therefore if a proton of energy $E_{0}$ produces (above a threshold energy $E_{\text {min }}$, and with a zenith angle dependence $\left.\propto(\cos \theta)^{-1}\right)$ an average number of muons $\left\langle N_{\mu}\left(E_{0}\right)\right\rangle_{p}$, a nucleus of mass number $A$ and the same total energy will produce with good approximation [5, 6] an average number of muons: $\left\langle N_{\mu}\left(E_{0}\right)\right\rangle_{A} \simeq A\left\langle N_{\mu}\left(E_{0} / A\right)\right\rangle_{p}$. The average muon multiplicity of a proton shower grows more slowly that the primary energy, approximately as $E^{0.75}$; this can be understood observing that in the approximation of Feynamn scaling the number of mesons above energy $E_{\text {min }}$ in a shower, neglecting threshold effects, grows linearly with energy, but with increasing energy the mesons are produced deeper in the atmosphere, where their decay is more rare. Therefore the average number of high energy muons in a shower of energy $E_{0}$ depends on the mass number of the primary nucleus approximately as $\propto A^{0.25}$, and (for the same energy spectrum) a primary comic ray flux rich in heavy nuclei will produce more high multiplicity events in deep undergroud muon detectors than a flux with a 'light' composition. This allows us in principle to obtain information on the primary cosmic ray spectrum and composition from underground measurements of multiple muon events. The uncertainty on the properties of the hadronic interactions is the main source of systematic errors in the development of this program.

In this work we will discuss some methods to estimate the size of this uncertainty. Elbert [7] has suggested that the average number of muons above a minimum energy $E_{\min }$ produced in the shower of a primary proton of energy $E_{0}$ has the scaling form:

$$
\left\langle N_{\mu}\left(E_{\min }, E_{0}\right)\right\rangle=\frac{1}{E_{\min } \cos \theta} G\left(\frac{E_{\min }}{E_{0}}\right),
$$

Gaisser and Stanev [8] and Forti et al. 9] have also fitted their montecarlo results with the form (回). In section 2 we will derive formally this result, discussing under which conditions it is valid, we will also show how it is possible to compute with analytic methods the function $G(x)$ from a knowledge of the inclusive single-particle differential cross sections.

In section 3 we will discuss a family of 'toy models' for the hadronic cross sections that are a generalization of the algorithms introduced by Hillas [10]. These models are fully described by a small set of simple montecarlo algorithms, and are therefore constructed as montecarlo instruments, at the same time the inclusive differential cross sections generated by the algorithms have simple closed form analytic representations, and are also suitable for analytic studies.

In section 4 we will discuss some explicit calculations of high energy muon production in proton showers, comparing analytic and montecarlo methods. The montecarlo 
technique is necessary for a correct treatment of fluctuations in the shower development.

In section 5 we will compare two models of hadronic interactions that are constructed to produce the same inclusive muon flux, with one model having a larger and softer $\pi$ multiplicity, and discuss how the multiplicity distribution of $\mathrm{TeV}$ muons changes.

\section{Analytic Method}

In this section we will discuss how under two approximations: (i) energy independent interaction lengths, (ii) validity of Feynman scaling in the fragmentation region of the hadronic cross sections, it is possible to solve analytically the shower development equations and calculate the inclusive high energy muon spectrum produced by a primary proton of energy $E_{0}$. The solution has the scaling form (四) suggested by Elbert [7] and used in [8, 9], (there is also a differential form of this scaling law given in equation (9)). The function $G(x)$ depends on the interactions lengths of nucleons, and mesons, and on the inclusive differential cross sections $d \sigma_{a \rightarrow b} / d x$ for production of particle $a$ in the interaction of particle $b$ with $x=E_{b} / E_{a}$. There is also a dependence on the density distribution of the medium where the showers develop. For an exponential distribution: $\rho(h) \propto e^{-h / h_{0}}$, as is the case for cosmic rays reaching the earth atmosphere and zenith angles not too close to the horizontal direction, this results in $G$ being proportional to the scale height $h_{0}$.

The analytic calculation of the functions $G$ can be performed with a straightforward generalization of the methods used for the calculation of electromagnetic showers [11]. We will begin to discuss the inclusive muon flux produced by a power law flux of primary particles, and then discuss the case of a monochromatic beam of particles.

\subsection{Power law initial proton spectrum}

The calculation of the inclusive muon flux produced by a power law primary flux, is a well known problem (see for example the textbook [5]), and is directly applicable to the real flux of primary particle reaching the earth. The all nucleon cosmic rays spectrum is in fact well represented by a power law: $\phi_{0}\left(E_{0}\right) \simeq K E_{0}^{-\alpha}$ with index $\alpha=2.7$, and a constant $K \simeq 1.85$ (with $E_{0}$ in $\mathrm{GeV}$ and $\phi$ in $\left.\left(\mathrm{cm}^{2} \mathrm{~s} \text { sr } \mathrm{GeV}\right)^{-1}\right)$. Using the approximations of constant interactions lengths and Feynman scaling, the muon spectrum above an energy of $\sim 20 \mathrm{GeV}$ has the approximate form [5]:

$$
\phi_{\mu}(E, \theta)=\left\{L_{\pi}(\alpha)\left[1+\frac{L_{\pi}(\alpha)}{H_{\pi}(\alpha)} \frac{E \cos \theta}{\varepsilon_{\pi}}\right]^{-1}+L_{K}(\alpha)\left[1+\frac{L_{K}(\alpha)}{H_{K}(\alpha)} \frac{E \cos \theta}{\varepsilon_{K}}\right]^{-1}\right\} K E^{-\alpha}
$$

We will be mostly interested in the high energy limit $\left(E \gg \varepsilon_{\pi}, \varepsilon_{K}\right)$ :

$$
\phi_{\mu}(E, \theta)=\frac{\varepsilon_{\pi} H_{\pi}(\alpha)+\varepsilon_{K} H_{K}(\alpha)}{E \cos \theta} K E^{-\alpha}=\frac{\epsilon_{\mu}(\alpha)}{E \cos \theta} K E^{-\alpha}
$$


The critical energy $\varepsilon_{\pi}=m_{\pi} h_{0} / c \tau_{\pi} \simeq 115 \mathrm{GeV}$ can be interpreted approximately as the energy at wich the average charged pion produced in a shower has a probability of $1 / 2$ to decay, $\varepsilon_{K} \simeq 850 \mathrm{GeV}$ has the same meaning for charged kaons, $m_{\pi}\left(m_{K}\right)$ and $\tau_{\pi}\left(\tau_{K}\right)$ are the mass and lifetime of a charged pion (kaon), $h_{0}=6.34 \mathrm{~km}$ is the scale-height of the stratosphere. The 'constants' $L_{\pi}, L_{K}, H_{\pi}$ and $H_{K}$, depend on the esponent of the primary flux $\alpha$, and on the properties of hadronic interactions. Explicit solutions for $L_{\pi}(\alpha)$ and $H_{\pi}(\alpha)$ are

$$
\begin{gathered}
L_{\pi}(\alpha)=Z_{N \pi}\left[\frac{1-r_{\pi}^{\alpha}}{\alpha\left(1-r_{\pi}\right)}\right] \\
H_{\pi}(\alpha)=\frac{Z_{N \pi}}{1-Z_{N N}} \frac{\Lambda_{\pi}}{\Lambda_{\pi}-\Lambda_{N}} \ln \left(\frac{\Lambda_{\pi}}{\Lambda_{N}}\right)\left[\frac{1-r_{\pi}^{\alpha+1}}{(\alpha+1)\left(1-r_{\pi}\right)}\right]
\end{gathered}
$$

In these equations: $r_{\pi}=\left(m_{\mu} / m_{\pi}\right)^{2}$; the quantities $Z_{a b}(\alpha)$ are defined as:

$$
Z_{a b}(\alpha)=\int_{0}^{1} d x x^{\alpha-1}\left(\frac{d n(x)}{d x}\right)_{a \rightarrow b},
$$

where $d n_{a \rightarrow b} / d x$ is the spectrum of particle $b$ in the interaction of particle $a$ with a target air nucleus and $x=E_{b} / E_{a} ; \Lambda_{N}=\lambda_{N} /\left(1-Z_{N N}\right), \Lambda_{\pi}=\lambda_{\pi} /\left(1-Z_{\pi \pi}\right)$ where $\lambda_{N}$ and $\lambda_{\pi}$ are the interactions lengths in air for nucleons and pions. The constants $L_{K}(\alpha)$ and $H_{K}(\alpha)$ have similar expressions, the inclusion of the processes $\left(\pi^{ \pm} \rightarrow K^{ \pm}\right)$and $\left(K_{L} \rightarrow K^{ \pm}\right)$ introduces some small complications [12]. The flux of only positive or negative muons can also be calculated with expressions similar to (2).

\subsection{Monochromatic initial proton spectrum}

The flux of muon produced by a monochromatic beam of protons takes a more complicated form. We can define the function $f\left(E ; E_{0}\right)$, the inclusive differential spectrum of $\mu$ 's produced by a primary proton of energy $E_{0}$, and $g\left(E_{\min } ; E_{0}\right)$ the integral spectrum (or average number of muons) above energy $E_{\text {min }}$. The functions $f$ and $g$ are related by:

$$
g\left(E_{\min } ; E_{0}\right) \equiv\left\langle N_{\mu}\left(E_{\min }, E_{0}\right)\right\rangle=\int_{E_{\min }}^{E_{0}} d E f\left(E ; E_{0}\right)
$$

If $E\left(E_{\min }\right) \gg \varepsilon_{\pi}, \varepsilon_{K}$, equation (3) implies:

$$
\left[\int_{E}^{\infty} d E_{0} E_{0}^{-\alpha} f\left(E, E_{0}\right)\right] E^{\alpha+1}=\epsilon_{\mu}(\alpha) \equiv \varepsilon_{\pi} H_{\pi}(\alpha)+\varepsilon_{K} H_{K}(\alpha)
$$

This condition can be satisfied only if the functions $f$ and $g$ have the scaling form :

$$
\begin{gathered}
f\left(E ; E_{0}\right)=\frac{1}{\cos \theta} \frac{1}{E^{2}} F\left(\frac{E}{E_{0}}\right) \\
g\left(E_{\min } ; E_{0}\right)=\frac{1}{\cos \theta} \frac{1}{E_{\min }} G\left(\frac{E_{\min }}{E_{0}}\right)
\end{gathered}
$$


Equation (8) defines also the Mellin transforms $M_{F}$ and $M_{G}$ of the function $F$ and $G$ :

$$
\begin{aligned}
& M_{F}(s)=\epsilon_{\mu}(s+2) \\
& M_{G}(s)=\frac{\epsilon_{\mu}(s+2)}{s+4}
\end{aligned}
$$

The Mellin transform of a functions $A(x)$ defined in the interval $0 \leq x \leq 1$ is:

$$
M_{A}(s)=\int_{0}^{1} d x x^{s} A(x)
$$

From equation (7) it is possible to deduce the following relation between the functions $F$ and $G$ :

$$
F(x)=-x^{2} \frac{d}{d x}\left[\frac{G(x)}{x}\right] .
$$

that implies in general $M_{G}(s)=M_{F}(s) /(s+4)$.

For continuous $A(x)$ the transform $M_{A}(s)$ is well defined in the complex field for all values of the imaginary part of its argument $\Im[s]$, and for the real part in the interval $s_{1} \leq \Re[s] \leq s_{2}$. The relation (13) can be inverted [13, [1] with:

$$
A(x)=\frac{1}{2 \pi i} \int_{C} d s M_{A}(s) x^{-i(s+1)}
$$

where the integral is taken in the complex field along an arbitrary path $C$ that starts from $\Im(s)=-\infty$ and ends at $\Im(s)=+\infty$, and is entirely contained in the domain of definition of $M_{A}(s)$.

To summarize: the function $\epsilon_{\mu}(\alpha)$, introduced in (3), for $\alpha$ real and positive determines the high energy inclusive muon flux produced by a power law primary flux of index $\alpha$ : $\phi_{\mu}(E) / \phi_{0}(E)=\epsilon_{\mu}(\alpha) /(E \cos \theta)$. The function $\epsilon_{\mu}(\alpha)$ can be calculated from the inclusive hadronic cross section according to equations (33) and (5), and the definition is valid also for $\alpha$ in an infinite region of the complex field. According to equations (11) and (12), $\epsilon_{\mu}(\alpha)$ gives also the Mellin transform of the functions $F(x)$ and $G(x)$ that determine the differential and integral inclusive muon spectrum for a monochromatic beam of protons. We can therefore compute the functions $G(x)$ and $F(x)$ in two steps: (i) compute the function $\epsilon_{\mu}(\alpha)$, (ii) use the inversion formula (15).

\section{Models for the differential cross sections}

In this section we will discuss an explicit family of models for the hadronic cross sections, that is a simple generalization of the montecarlo algorithms proposed by Hillas [10], to model multiple particle production in high energy hadronic interactions. These algorithms, in spite of their simplicity, give results in good agreement with data. The inclusive differential cross sections generated by the montecarlo algorithms have also ex-

plicit and exact analytic representations, this allows to cross check results obtained with analytic and montecarlo methods. 
To explore the sensitivity of muon production to the characteristics of the cross sections, we have generalised the set of algorithms originally proposed by Hillas, introducing some parameters that can be changed continuously. In chapter 17 of reference [5], Gaisser describes in some detail the Hillas algorithms, and discuss also possible generalizations. In [10] Hillas considered only nucleons and pions, neglecting kaons and other particles. It is rather straightforward in the framework of the model to include kaons or treat separately protons and neutrons, but for the sake of simplicity we will also consider only nucleons and pions. The separate treatment of protons and neutrons is of little importance if we sum over the charge of the muons; only $\sim 25 \%$ of $\mathrm{TeV}$ muons are produced by kaons, and therefore, for our illustrative discussion, kaons are not of crucial importance. The main focus of this work is not to obtain an absolute calculation but to discuss the sensitivity of the results to variations of the 'input'. The algorithms that we are considering are exactly scaling in the variable $x=E / E_{0}$ where $E_{0}$ is the incident particle energy. Scaling violations can be be introduced allowing the parameters of the model to vary with energy, we will however discuss here only scaling models, and concentrate on the study of the effects of distortions of the spectra of nucleons and pions. We will not discuss the transverse momentum of the particles produced in the shower, and we will not comment on the separation between muons.

\subsection{Proton interactions}

The set of algorithms to generate the interaction of a proton of initial energyy $E_{0}$ are:

1. Generate $x(0 \leq x \leq 1)$ with linear distribution of average $\langle x\rangle=1-K_{p}$. Construct a leading nucleon of energy $E_{0} x$.

2. The remaining energy $E_{0}(1-x)$ is divided into two parts $A$ and $B$ with a flat distribution. Then each piece of energy is split again into two parts with a flat distribution $\left(A \rightarrow A_{1}+A_{2}\right.$ and $\left.B \rightarrow B_{1}+B_{2}\right)$.

3. Each of the four pieces of energy has now a probability $P^{*}$ of splitting once again into two parts, always with flat distribution.

4. At this point we have $N$ energy pieces $(4 \leq N \leq 8)$, with a binomial probability distribution $p(N)=P_{\text {binomial }}\left(N-4 ; 4, P^{*}\right)$. Each piece of energy now enters a recursive process.

5. The energy of the piece is splitted with flat distribution into two parts. A part is assigned as the energy of a pion (with equal probability for the three charges), the remaining energy is again divided into two parts: one is assigned to a new pion, for the other the process is iterated. The splitting is stopped when the remaining energy is less than a pion mass or of some preassigned threshold value.

In the algorithms we have introduced two parameters: the inelasticity $K_{p}\left(1 / 2 \leq K_{p} \leq\right.$ $2 / 3$ ) that gives the fraction of the projectile particle energy that is not transfered to the 
leading nucleon, and $P^{*}\left(0 \leq P^{*} \leq 1\right)$ that determines the shape of the pion spectrum, when $(x \rightarrow 1)$ the pion spectrum has the behaviour $\propto(1-x)^{3}$ if $P^{*}=0$ and $\propto(1-x)^{4}$ if $P^{*}=1$. A 'model' in this framework is then represented by the pair of numbers $\left\{K_{p}, P^{*}\right\}$. The original algorithms of Hillas corresponds to the model $\left\{\frac{1}{2}, 0\right\}$.

The montecarlo algorithms described above give inclusive differential cross sections that have explicit analytic expressions:

$$
\begin{gathered}
\frac{d n_{p p}}{d x}=1+6\left(K_{p}-\frac{1}{2}\right)-12\left(K_{p}-\frac{1}{2}\right) x \\
\frac{d n_{p \pi^{ \pm}}}{d x}=\frac{8}{3}\left[1-6\left(K_{p}-\frac{1}{2}\right)\right]\left\{\left[\frac{1}{x}-1+\ln x-\frac{(\ln x)^{2}}{2}\right]\left(1+P^{*}\right)+2 P^{*} \frac{(\log x)^{3}}{6}\right\}+ \\
+\frac{96}{3}\left(K_{p}-\frac{1}{2}\right)\left[\left(\frac{1}{2 x}-\frac{x}{2}+\ln x\right)\left(1-P^{*}\right)+2 P^{*}\left(\frac{1}{2 x}-1+\frac{x}{2}-\frac{(\ln x)^{2}}{2}\right)\right](1
\end{gathered}
$$

The differential cross section for $\pi^{\circ}$ production is simply $1 / 2$ of (17). The plateau of the rapidity distribution for the charged pions has a height that increases linearly with $\left(1+P^{*}\right)$ :

$$
\rho_{p \rightarrow \pi^{ \pm}} \equiv\left(\frac{d n_{p \pi^{ \pm}}}{d y}\right)_{y^{*}=0}=\frac{8}{3}\left[1+P^{*}\right]
$$

The momenta of the inclusive spectra $Z_{p X}(\alpha)=\left\langle x^{\alpha-1}\right\rangle_{p X}$ can be calculated explicitely:

$$
\begin{gathered}
Z_{p p}(\alpha)=\left[1+6\left(K_{p}-\frac{1}{2}\right)\right] \frac{1}{\alpha}-12\left(K_{p}-\frac{1}{2}\right) \frac{1}{\alpha+1} \\
Z_{p \pi^{ \pm}}(\alpha)=\frac{8}{3}\left[1-P^{*}\left(\frac{\alpha-2}{\alpha}\right)\right]\left[\frac{1}{\alpha^{3}(\alpha-1)}+\left(K_{p}-\frac{1}{2}\right) \frac{1}{\alpha^{2}}\left(\frac{12}{\alpha^{2}-1}-\frac{6}{\alpha}\right)\right]
\end{gathered}
$$

For $\alpha=2, Z_{p p}=1-K_{p}$ and $Z_{p \pi^{ \pm}}=\frac{2}{3} K_{p}$ independently from $P^{*}$ and the shape of the spectra.

\subsection{Charged pion interactions}

To generate a $\pi^{ \pm}$interaction the algorithms are the following

1. The total energy is divided into two parts $A$ and $B$ with a flat distribution. The first part $A$ is assigned to a pion with probability $P_{D}$. This 'diffractive' pion is charged with a probability of 0.87 , the probability of being of same (opposite) sign with respect to the projectile is $0.80(0.07)$.

2. The energy of the piece $B$ is divided into two parts $B_{1}$ and $B_{2}$, then each piece is again divided into two. We now have 4 energy pieces. $B_{11}, B_{12}, B_{21}$ and $B_{22}$.

3. Piece $A$ if it was not already assigned to the diffractive pion, is treated in the same way as piece $B$ in the previous and following steps. 
4. The pieces $B_{11}$ and $B_{21}$ have now each a probability $P_{A}$ of being assigned to a pion (with equal probability for the three charges).

5. Each of the remaining energy pieces $\left(B_{12}, B_{22}\right.$ and $B_{11}, B_{21}$ if not already assigned to pions) are splitted into two parts with flat distribution with a probability $P_{B}$

6. Part $B$ is now divided into $N(2 \leq N \leq 8)$ energy pieces. Each of these pieces is fragmented into particles, with a recursive process (as in proton interactions). The energy of one piece is splitted with flat distribution into two parts, a part is assigned as the energy of a pion, for the remaining energy the process is iterated until we are left with a remaining energy less than a pion mass or a preassigned threshold value.

The pion interaction model depends on three parameters $\left\{P_{D}, P_{A}, P_{B}\right\}$. The original algorithm proposed by Hillas [10] corresponds to the choice $\left\{\frac{1}{2}, \frac{1}{2}, 0\right\}$. The physical meaning of the parameters is easy to understand intuitively. Increasing $P_{D}$ and $P_{A}$ the pion spectrum is hardened and the multiplicity decreased. Increasing $P_{B}$ has the opposite effect: the spectrum is softened, and the multiplicity increases.

The inclusive pion spectrum implied by this set of algorithms can be calculated:

$$
\begin{aligned}
\frac{d n_{\pi^{ \pm} \pi^{ \pm}}}{d x} & =0.87 P_{D}+\frac{4}{3}\left(2-P_{D}\right) P_{A} \frac{(\ln x)^{2}}{2}+ \\
& +\frac{4}{3}\left(2-P_{A}\right)\left(2-P_{D}\right)\left[\left(\frac{1}{x}-1+\ln x-\frac{(\ln x)^{2}}{2}\right)\left(1+P_{B}\right)+2 P_{B} \frac{(\ln x)^{3}}{6}\right]
\end{aligned}
$$

The spectrum of $\pi^{\circ}$ is obtained changing the numerical coefficients of the three terms in (21): $0.87 \rightarrow 0.13,4 / 3 \rightarrow 2 / 3$, and $4 / 3 \rightarrow 2 / 3$. The momenta of the energy distribution are:

$$
Z_{\pi \pi^{ \pm}}=\frac{0.87 P_{D}}{\alpha}+\frac{4}{3}\left(2-P_{D}\right) P_{A} \frac{1}{\alpha^{3}}+\frac{4}{3}\left(2-P_{A}\right)\left(2-P_{D}\right) \frac{1}{\alpha^{3}(\alpha-1)}\left[1+\frac{2 P_{B}}{\alpha}\right]
$$

The rapidity density is:

$$
\rho_{\pi \pi}=\frac{4}{3}\left(2-P_{A}\right)\left(2-P_{D}\right)\left(1+P_{B}\right)
$$

\section{Explicit Calculations}

In this section we will discuss some calculations of $\mathrm{TeV}$ muons, using the algorithms discussed in the previous section to describe hadronic interactions. We will always assume that the interactions length are constant and that Feynman scaling is valid. In our framework therefore an 'hadronic interaction model' is fully described as a 'vector' of eight numbers: $M=\left\{\lambda_{p}, \lambda_{\pi} ; K_{p}, P^{*} ; P_{D}, P_{A}, P_{B}\right\}$, the first two quantities are the interactions lengths of nucleons and pions, the next two numbers describe protons interactions, the last three numbers describe pion interactions. 
The first quantity that is interesting to calculate is the inclusive high energy muon flux. This flux is determined by the quantity $\epsilon_{\mu}(\alpha)$ for $\alpha=2.7$. In table 1 we show the value of $\epsilon_{\mu}(2.7)$ calculated for different 'hadronic interaction models'.

Table 1. $\epsilon_{\mu}(\alpha=2.7)$. The primary flux has form: $\phi_{0}(E)=K E^{-2.7}$; the muon flux $(E \gg 100 \mathrm{GeV})$ has form: $\phi_{\mu}(E, \theta)=\epsilon_{\mu} /(E \cos \theta) \times \phi_{0}(E)$.

\begin{tabular}{|c|c||c|c||c|c|c||c|}
\hline$\lambda_{p}\left(\mathrm{~g} \mathrm{~cm}^{-2}\right)$ & $\lambda_{\pi}\left(\mathrm{g} \mathrm{cm}^{-2}\right)$ & $K_{p}$ & $P^{*}$ & $P_{D}$ & $P_{A}$ & $P_{B}$ & $\epsilon_{\mu}(\mathrm{GeV})$ \\
\hline \hline 86.0 & 111.8 & $1 / 2$ & 0 & $1 / 2$ & $1 / 2$ & 0 & 8.57 \\
\hline 77.4 & 111.8 & $1 / 2$ & 0 & $1 / 2$ & $1 / 2$ & 0 & 9.02 \\
86.0 & 100.6 & $1 / 2$ & 0 & $1 / 2$ & $1 / 2$ & 0 & 8.14 \\
77.4 & 100.6 & $1 / 2$ & 0 & $1 / 2$ & $1 / 2$ & 0 & 8.57 \\
\hline 86.0 & 111.8 & $1 / 3$ & 0 & $1 / 2$ & $1 / 2$ & 0 & 5.42 \\
86.0 & 111.8 & $2 / 3$ & 0 & $1 / 2$ & $1 / 2$ & 0 & 11.03 \\
86.0 & 111.8 & $1 / 2$ & 1 & $1 / 2$ & $1 / 2$ & 0 & 6.35 \\
\hline 86.0 & 111.8 & $1 / 2$ & 0 & 0 & $1 / 2$ & 0 & 7.96 \\
86.0 & 111.8 & $1 / 2$ & 0 & 1 & $1 / 2$ & 0 & 9.34 \\
86.0 & 111.8 & $1 / 2$ & 0 & $1 / 2$ & 0 & 0 & 8.45 \\
86.0 & 111.8 & $1 / 2$ & 0 & $1 / 2$ & 1 & 0 & 8.70 \\
86.0 & 111.8 & $1 / 2$ & 0 & $1 / 2$ & $1 / 2$ & 1 & 8.44 \\
\hline
\end{tabular}

The first line of table 1 corresponds to the differential cross sections proposed originally by Hillas [10], and is also our choice for a 'reference model', the other lines show the sensitivity to $10 \%$ changements in the interactions length, and to the maximum allowed variations of each of the parameters that we have constructed to describe the shape of the differential cross section. We can observe that the inclusive muon production is especially sensitive to the properties of the proton interactions. Decreasing the inelasticity $K_{p}$ or softening the pion spectrum (increasing $P^{*}$ ) depresses the muon flux. Modifications of the pion differential cross section have a smaller effect on the inclusive muon flux. In fact, because of the steepness of the primary flux, most of the muons are produced in the decay of mesons produced in the first interaction of a primary cosmic ray.

The function $G(x)$ that gives the average number of high energy muons produced by a primary particle is easily calculated from (12) using the inversion formula (15). In the models we are considering $\epsilon_{\mu}(\alpha)$ can be obtained from (3), (5), (19), (20), (22), as a simple combination of elementary functions, and the Mellin transform can be inverted with an easy numerical integration.

The function $G(x)$ calculated as discussed above for the 'reference model': [ $\lambda_{p}=86$ and $\left.\lambda_{\pi}=111.8 \mathrm{~g} \mathrm{~cm}^{-2},\left\{K_{p}, P^{*} ; P_{D}, P_{A}, P_{B}\right\}=\left\{\frac{1}{2}, 0 ; \frac{1}{2}, \frac{1}{2}, 0\right\}\right]$, is shown in figure 1 . In the same figure we also show for comparison the curves that Gaisser and Stanev [8] and Forti et al. 9] have used as fit to their montecarlo calculations. The agreement is surprisingly good considering the extreme simplicity of the model we are discussing. It should also be noted that the results of [8, 9] refer to muons not above a fixed threshold energy, but at a fixed depth $h$. For the comparison we have used the approximation 
$E_{\min }(h) \simeq 0.53\left(e^{0.4 h}-1\right)(h$ in km.w.e., $E$ in $\mathrm{TeV})$, fluctuations in the muon energy loss [14] should however be taken into account for a more detailed comparison.

In figures $2,3,4,5$ we show how the function $G(x)$ is modified because of changements in the shape and normalization of the inclusive differential cross sections. We have recalculated $G(x)$ using different values of the parameters. We will consider the 'reference model', and change one parameter at the time.

In figure $2 \mathrm{a}$ we show the $\left(p \rightarrow \pi^{ \pm}\right)$spectrum obtained with different values of the inelasticity $K_{p}=\frac{1}{2}, \frac{1}{3}, \frac{2}{3}$ and $P^{*}=0$. In figure $2 \mathrm{~b}$, we show the function $G(x)$ as a function of $1 / x=E_{0} / E_{\min }$ for these three values of $K_{p}$. As expected the number of muons increases for larger $K_{p}$, because more energy is transfered to pions. With increasing $E_{0} / E_{\min }$ however the difference becomes smaller, the curves join, and in fact on close inspection cross each other. This can be explained observing that for large $E_{0} / E_{\min }$ it is possible to obtain more pions above the threshold energy $E_{\min }$ giving more energy to the leading nucleon in the interaction. The number of pions in the first interaction of the shower decreases, but additional pions are created in the second interaction, our calculation takes into account the fact that these additional pions are produced deeper in the atmosphere and have a smaller probability to decay.

In figure $3 \mathrm{a}$ we show the $\left(p \rightarrow \pi^{ \pm}\right)$spectrum for $P^{*}=1,0\left(K_{p}=\frac{1}{2}\right)$. As discussed before $P^{*}=1$ corresponds to a softer spectrum, with a higher multiplicity. In figure $3 \mathrm{~b}$ we show the function $G(x)$ as a function of $1 / x=E_{0} / E_{\min }$ calculated for the two pion spectra. For small $E_{0} / E_{\min }$ the curve corresponding to $P^{*}=0$ (harder $\pi$ spectrum) is higher; the two curves cross each other at $E_{0} / E_{\min } \simeq 30$. This is qualitatively easy to understand: for small $E_{0} / E_{\min }$ the harder spectrum produces more pions above threshold, when $E_{0} / E_{\min }$ grows, the effect of the larger pion multiplicity becomes dominant.

In figure $4 \mathrm{a}$ and $5 \mathrm{a}$ we show the $\left(\pi^{ \pm} \rightarrow \pi^{ \pm}\right)$spectrum for different values of the parameters : $P_{D}=0, \frac{1}{2}, 1$, and $P_{B}=0,1$. In figure $4 \mathrm{~b}$ and $5 \mathrm{~b}$ we show the curve $G(x)$ calculated with the different spectra. Some remarks are : (i) very large deformation of the $\left(\pi^{ \pm} \rightarrow \pi^{ \pm}\right)$spectrum result in small variations of $\left\langle N_{\mu}\left(E_{\min }, E_{0}\right)\right\rangle$; (ii) the effect is very small for $E_{0} / E_{\min } \lesssim 10$ when most muons come from the decay of first generation mesons; (iii) the largest effects comes from different treatments of $\pi$ diffraction (fig $4 \mathrm{~b}$ ); (iv) a softening of the $\pi \rightarrow \pi$ spectrum from $d n / d x \propto(1-x)^{3}\left(P_{B}=0\right)$ to $\propto(1-x)^{4}$ $\left(P_{B}=1\right)$ depresses muon production for $E_{0} / E_{\min } \lesssim 10^{3}$, then enhances it. The effect (see fig $5 \mathrm{~b})$ is however small.

To summarize the information of the effect of $G(x)$ of the changement of the parameters of the model, it can be useful to discuss the quantity $\xi_{P}\left(E_{\min } / E_{0}\right)$, the logarithm derivative of the average muon multiplicity as a function of the parameter $P$ taken from the 'starting model' that we take as the original Hillas model: $\left\{K_{p}, P^{*} ; P_{D}, P_{A}, P_{B}\right\}=\left\{\frac{1}{2}, 0, \frac{1}{2}, \frac{1}{2}, 0\right\}$ with $\lambda_{p}=86$ and $\lambda_{\pi}=111.8 \mathrm{~g} \mathrm{~cm}^{-2}$.

$$
\xi_{P}\left(E_{\min } / E_{0}\right)=\frac{\partial \log \left\langle N_{\mu}\left(E_{\min }, E_{0}\right)\right\rangle}{\partial \log P} \simeq \frac{\Delta\left\langle N_{\mu}\right\rangle}{\left\langle N_{\mu}\right\rangle}\left(\frac{\Delta P}{P}\right)^{-1}
$$

The meaning of $\xi_{P}$ is that if the parameter $P$ is changed by (for example) $10 \%$, the 
resulting percentual effect on $\left\langle N_{\mu}\left(E_{\min }, E_{0}\right)\right\rangle$ is $\left(\xi_{P} \times 10\right) \%$. A positive (negative) $\xi_{P}$ indicates that an increase of parameter $P$ will produce a depression (enhancement) of $\left\langle N_{\mu}\right\rangle$. The logarithimic derivatives $\xi_{P}(x)$ are shown in table 2.

Table 2. Logarithmic derivatives $\xi_{P}(\mathrm{x}) .\left(x=E_{\min } / E_{0}\right)$.

\begin{tabular}{|c||r|r|r|r|r|}
\hline Parameter $/ x$ & $10^{-1}$ & $10^{-2}$ & $10^{-3}$ & $10^{-4}$ & $10^{-5}$ \\
\hline$\lambda_{p}$ & -0.530 & -0.497 & -0.411 & -0.333 & -0.276 \\
$\lambda_{\pi}$ & 0.512 & 0.483 & 0.405 & 0.333 & 0.280 \\
$K_{p}$ & 0.985 & 0.230 & 0.082 & 0.033 & -0.002 \\
$\left(1-P^{*}\right)$ & -0.282 & 0.133 & 0.192 & 0.173 & 0.155 \\
$P_{D}$ & 0.082 & 0.107 & 0.077 & 0.041 & 0.012 \\
$P_{A}$ & 0.012 & 0.033 & 0.004 & -0.043 & -0.088 \\
$\left(1-P_{B}\right)$ & -0.009 & -0.053 & -0.025 & 0.054 & 0.131 \\
\hline
\end{tabular}

The first two lines of table 2 , show the dependence on the hadronic interaction lengths. An increase in $\lambda_{p}$ decrease the number of muons because with a larger proton interaction lengths the shower develops deeper in the atmosphere where the density is higher and muons decay is more difficult. When $E_{0} / E_{\text {min }}$ grows the effect of a changement of $\lambda_{p}$ decreases in importance because a growing fraction the muons is produced in a cascade of type $p \rightarrow \pi \rightarrow \pi \rightarrow \mu$.

An increase of $\lambda_{\pi}$ increases the number of $\mathrm{TeV}$ muons because if the interactions length is longer the pions have more time to decay. The effect becomes smaller with increasing $E_{0} / E_{\text {min }}$, because a larger $\lambda_{\pi}$ will also produce a deeper shower, and the pions produced in a cascade of type $p \rightarrow \pi \rightarrow \pi$ are created at lower altitude and decay more rarely. Note that the two hadronic interaction lengths enter in the expressions for muon production only in the combination: $\Lambda_{\pi} / \Lambda_{p} \simeq \lambda_{\pi} / \lambda_{p}$ and therefore $\xi\left(\lambda_{\pi}\right) \simeq-\xi\left(\lambda_{p}\right)$.

The other lines in table 2 describe the dependence on the differential cross sections, the same comments developed for the discussion of figures 2-5 apply, one may notice the changements of sign of $\xi\left(K_{p}\right)$ (at very large $E_{0} / E_{\min }$ ) and of $\xi\left(P^{*}\right)$. It is encouraging to see that $\left|\xi_{P}\right|$ is always less than 1 , showing that the sensitivity to distortions of the spectral shape is only moderate. Note how $G(x)$ is relatively insensitive to the properties of pion interactions.

\subsection{Fluctuations}

The analytic method that we have described allows us to compute the average number of high energy muons in a shower, but does not take into account fluctuations in the shower development. In order to study the importance of fluctuations and also to check the analytic calculation we have prepared a montecarlo implementation of the family of interaction models discussed in section 3. The straightforward montecarlo method is based of the shower code developed in Bartol [15] (see also [5]) and is based on the following steps: (i) a primary cosmic ray is propagated in the atmosphere until it interacts; (ii) 
a set of secondary particles (pions and nucleons) are produced at the interaction point according to the algorithms described in section 3; (iii) each one of the secondary particles is propagated until it interacts or decay; (iv) at each decay or interaction vertex the incident particle is destroyed and a set of new particles is produced conserving energy and momentum; (v) the procedure is iterated until all particles are below a preassigned minimum energy. All produced muons are recorded.

In figure 6 we show the inclusive muon spectrum above $1 \mathrm{TeV}$ produced by a vertical primary proton of energy $10,10^{2}, 10^{3}$ and $10^{4} \mathrm{TeV}$, obtained with the montecarlo method and with the analytic formula using our 'reference model'. The results obtained with the two methods are in excellent agreement between each other. The montecarlo method allows to study not only $\left\langle N_{\mu}\right\rangle$ but also the probability distribution $P\left(N_{\mu}\right)$ of having exactly $N_{\mu}$ muons in a shower. The distributions $P\left(N_{\mu}\right)$ calculated with the montecarlo method for $E_{0}=10$ and $10^{4} \mathrm{TeV}\left(E_{\mu}^{\mathrm{min}}=1 \mathrm{TeV}, \theta=0^{\circ}\right)$ are shown in figure $7 \mathrm{a}$ and $7 \mathrm{~b}$. In the same figures we compare the montecarlo results with a poissonian distribution of the same average and a negative binomial distribution of same average and dispersion. The distribution $P\left(N_{\mu}\right)$ is broader than a poissonian, and the difference becomes more marked with increasing energy, the negative-binomial being a good fit. These results have been found previoulsy by Forti and collaborators [9]; we would like to stress that the nonpoissonian fluctuations are not connected to violations of Feynman or KNO scaling, and are present also in exactly scaling models as those we are discussing.

\section{$5 \quad$ Interaction Model and Multiplicity Distribution}

As an illustration of the importance of the interaction model for the calculation of the multiplicity distribution of underground muons, we have calculated the fluxes of $\mathrm{TeV}$ muons using a 'realistic' proton flux of energy spectrum: $\phi_{0}(E)=1.85 E^{-2.7}$, steepening to $\propto E^{-3}$ for $E \geq 3 \times 10^{6} \mathrm{GeV}\left(E\right.$ in $\mathrm{GeV}$ and $\phi$ in $\left.\left.\left(\mathrm{cm}^{2} \mathrm{~s} \mathrm{sr}\right)^{-1}\right)\right)$, and two different models for the hadronci interactions. In both models $\lambda_{p}=86$ and $\lambda_{\pi}=111.8 \mathrm{~g} \mathrm{~cm}^{-2}$, and the charged pion interactions: are described by the algorithms originally proposed by Hillas with parameters $\left\{P_{D}, P_{A}, P_{B}\right\}=\left\{\frac{1}{2}, \frac{1}{2}, 0\right\}$. The two models differ in the treatment of proton interactions. The first model is nearly identical to the 'reference model' with parameters: $\left\{K_{p}, P^{*}\right\}=\{0.475,0\}$; the second model has a larger inelasticity but a softer spectrum: $\left\{K_{p}, P^{*}\right\}=\left\{\frac{2}{3}, 1\right\}$. The $G$ functions obtained with the two models is shown in figure 8 , both models have $\epsilon_{\mu}(2.7)=4.7 M_{G}(0.7) \simeq 8.16 \mathrm{GeV}$, and for energies $E \gg \varepsilon_{\pi}$ produce essentially identical 'inclusive' muon fluxes. The larger multiplicity of model-2 exactly compensates its softer spectrum. We may however expect that using model-2 the probability of having several muons in the same shower is larger.

To investigate quantitatively this possibility we have generated approximately 3 million vertical showers with $E_{0} \geq 1 \mathrm{TeV}$ for each of the two models. In order to increase the statistics of events with high muon multiplicity we have sampled the energy of the showers from a distribution $\propto E_{0}^{-1.75}$, weighting each event with $E^{1.75} / \phi_{0}(E)$. In figure 9 we show the obtained inclusive muon fluxes that are essentially identical in the two 
models, and very well represented by: $\phi_{\mu}(E)=\epsilon / E \phi_{0}(E)$ with $\epsilon=8.16 \mathrm{GeV}$. The muon multiplicity $\left(E_{\mu} \geq 1 \mathrm{TeV}\right)$ for the two models is shown in figure 10a, and the ratio of the fluxes obtained the two models for the same multiplicity is shown in figure 10b. The inclusive fluxes $\phi_{1}+2 \phi_{2}+\ldots+n \phi_{n}+\ldots$ are equal to better that $1 \%$, but the flux of single muons is $5.5 \%$ smaller using model- 2 , the ratio model $-2 /$ model- 1 becomes 1.22 for double muons, grows to 1.46 for triples, to 1.76 for quadruple muons, and then seems to remain approximately constant.

The two models considered are 'physically consistent', both respect conservation laws (energy, momentum, baryon number), and both produce the same inclusive muon flux, however the same experimental multiplicity distribution of underground muons if interpreted with model-1 (model-2) would result in a heavier (lighter) composition, because the effects of the smaller (larger) frequency of high multiplicity events should be compensated with a different mass distribution of the primaries.

In this work we do not attempt a more realistic and complete discussion, that will be presented in a future paper. We note that the fairly extreme distortions of the spectra that we have tried, produce a difference of about a factor of 2 for the frequency of events with multiplicity $\gtrsim 10$. The present range of uncertainty in composition [3] can produce larger differences.

\section{Conclusions}

In this work we have formally derived the result that the average number of high energy muons $\left(E_{\min } \gtrsim 1 \mathrm{TeV}\right)$ produced by a primary cosmic ray proton of energy $E_{0}$ has the scaling form: $\left\langle N_{\mu}\left(E_{\min }, E_{0}\right)\right\rangle=G\left(E_{\min } / E_{0}\right) / E_{\min }$. The function $G(x)$ is calculable analytically from a knowledge of the inclusive single-particle differential cross sections. We have illustrated how the shape and normalization of $G(x)$ depends on the detailed form of these cross sections.

We do expect detectable deviations from the scaling behaviour. A source of deviation is simply the fact that the critical energy for kaon decay $\varepsilon_{K}$ is not small with respect to $1 \mathrm{TeV}$. A second source of deviation is due to the fact that the hadronic interactions lengths are decreasing with energy. There is also the possibility of observable violations of Feynman scaling in the fragmentation region, the measured growth of the central plateau should have visible effects for large $E_{0} / E_{\text {min }}$.

We have discussed a possible generalization of the montecarlo algorithms originally developed by Hillas [10], to describe the properties of of hadronic interactions. These algorithms because of their remarkable simplicity and flexibility, can be a useful too to study in detail the effects of uncertainties in the properties of hadronic interactions in the development of showers. They could be very useful for the study of the highest energy cosmic rays $\left(E \sim 10^{20} \mathrm{eV}\right)$.

Uncertainties in the modeling of hadronic interactions are the dominant source of systematic error for the measurement of cosmic ray composition from data on multiple muon events in deep underground detectors. The spectrum of the leading nucleon, and 
of fast pions produced in nucleon interactions are of special importance. The details of particle production in pion interactions are less important to control.

\section{Acknowledgments}

Part of this work was developed during a visit to the Bartol Research Center whose hospitality is gratefully acknowledged. Many of the ideas presented in this paper were formed in conversations with T.K. Gaisser and T.Stanev. I'm also grateful to Giuseppe Battistoni, Sergio Petrera and Ornella Palamara for many interesting discussions on the problem of underground muons, and to M.Hillas for useful clarifications. T.K Gaisser kindly read and commented an early version of this paper. 


\section{Figure Captions}

Fig. 1. Curve $G(x)$ (equation (1)) calculated with the Hillas model. The curve is compared with the parametrization of the average number of muon at depth $h$ of Gaisser and Stanev [8] and Forti et al. [9] assuming the approximate relation $E_{\text {min }}=0.53\left(e^{0.4 h}-1\right)(E$ in $\mathrm{TeV}, h$ in km.w.e. $)$.

Fig. 2a. Plot of the inclusive $\left(p \rightarrow \pi^{ \pm}\right)$spectrum calculated with $P^{*}=0$ and with inelasticity $K_{p}=\frac{1}{2}, \frac{1}{3}, \frac{2}{3}$.

Fig. 2b. Curve $G(x)$ calculated with the 'reference model' $\left[\left\{K_{p}, P^{*} ; P_{D}, P_{A}, P_{B}\right\}=\right.$ $\left.\left\{\frac{1}{2}, 0, \frac{1}{2}, \frac{1}{2}, 0\right\}, \lambda_{p}=86, \lambda_{\pi}=111.8 \mathrm{~g} \mathrm{~cm}^{-2}\right]$ and with modified inelasticity $K_{p}=\frac{1}{3}$, $\frac{2}{3}$.

Fig. 3a. Plot of the inclusive $\left(p \rightarrow \pi^{ \pm}\right)$spectrum calculated with $P^{*}=0$ and $P^{*}=1\left(K_{p}=\frac{1}{2}\right)$.

Fig. 3b. Curve $G(x)$ calculated with the 'reference model' $\left(P^{*}=1\right)$ and with the modification $P^{*}=1$.

Fig. 4a. Plot of the inclusive $\left(\pi^{ \pm} \rightarrow \pi^{ \pm}\right)$spectrum calculated with the model $\left\{P_{D}, P_{A}, P_{B}\right\}=\left\{P_{D}, \frac{1}{2}, 0\right\}$ and $P_{D}=0, \frac{1}{2}, 1$.

Fig. 4b. Curve $G(x)$ calculated with the 'reference model' $\left(P_{D}=\frac{1}{2}\right)$ and with $P_{D}=0,1$.

Fig. 5a. Plot of the inclusive $\left(\pi^{ \pm} \rightarrow \pi^{ \pm}\right)$spectrum calculated with the model $\left\{P_{D}, P_{A}, P_{B}\right\}=\left\{\frac{1}{2}, \frac{1}{2}, P_{B}\right\}$ and $P_{B}=0,1$.

Fig. 5b. Curve $G(x)$ calculated with the 'reference model' $\left(P_{B}=0\right)$ and with $P_{B}=1$.

Fig. 6. Plot of $f\left(E_{\mu} ; E_{0}\right)$ the inclusive differential muon spectrum produced by a vertical primary proton for $E_{0}=10,100,10^{3}$ and $10^{4} \mathrm{TeV}$. The interaction model used is the 'reference model'. The curves are analytic calculations, the histograms the results of Montecarlo runs.

Fig. 7a. Probability distribution $P\left(N_{\mu} ; E_{0}, E_{\min }\right)$ that a primary proton of energy $E_{0}$ produces $N_{\mu}$ muons above threshold energy $E_{\min }$. The points are the results of Montecarlo calculation with $\theta=0^{\circ}, E_{\min }=1 \mathrm{TeV}, E_{0}=10^{2} \mathrm{TeV}$. The dashed curve is a poissonian distribution with the same average, the solid line is a negativebinomial distribution of same average and dispersion, as the montecarlo result.

Fig. 7b. As in figure $7 \mathrm{a}, E_{0}=10^{4} \mathrm{TeV}$. 
Fig. 8. Function $\mathrm{G}(\mathrm{x})$ for two models that yield the same inclusive muon distribution. The proton interactions are described by $\left\{K_{p}, P^{*}\right\}=\{0.475,0\}$ in model-1 and $\left\{\frac{2}{3}, 1\right\}$ in model-2. The other parameters are chosen as in the 'reference model'.

Fig. 9. Inclusive muon flux calculated with analytic and montecarlo methods assuming the primary proton flux $\phi_{0}(E)=1.85 E^{-2.7}$ steepening to $\propto E^{-3}$ for $E \geq 3 \times 10^{6} \mathrm{GeV}$. (see text).

Fig. 10a. Muon multiplicity distribution $\phi_{\mu}\left(N_{\mu}\right)\left(E_{\min }=1 \mathrm{TeV}\right.$ and $\left.\theta=0^{\circ}\right)$ calculated with a montecarlo method assuming a primary cosmic ray flux of protons with energy spectrum: $\phi_{0}(E)=1.85 E^{-2.7}$ steepening to $\propto E^{-3}$ for $E \geq 3 \times$ $10^{6} \mathrm{GeV}$. The two set of points refer to two different proton interaction models : $\left\{K_{p}, P^{*}\right\}=\{0.475,0\}$ and $\left\{K_{p}, P^{*}\right\}=\left\{\frac{2}{3}, 1\right\}$ that result in the same inclusive muon distribution.

Fig. 10b. Ratio of the fluxes $\phi_{\mu}\left(N_{\mu}\right)$ of figure $9 \mathrm{a}$, calculated with two different models for proton iteractions. 


\section{References}

[1] Ch.Berger et al. (Fréjus collaboration) Phys.Rev. D 40, 2163, (1989)

[2] M.Aglietta et al. (NUSEX collaboration), Proc. of XXI ICRC conference, Adelaide, Australia (1990), paper HE 4.5-1, vol.9 pag 352.

[3] S.Ahlen et al. (MACRO collaboration) Phys.Rev. D 46, 895, (1992).

[4] S.Ahlen et al. (MACRO collaboration) Phys.Rev. D 46, 4836, (1992).

[5] T.K.Gaisser, "Cosmic Rays and Particle Physics", Cambridge University Press, (1990).

[6] J.Engel, T.K.Gaisser, P.Lipari and T.Stanev, Phys.Rev. D 46, 5013, (1992).

[7] J.W.Elbert, Proc. Dumand Summer Workshop, La Jolla, California, ed. A.Roberts (Scripps Institution of Oceanography, La Jolla, 1979), vol.2, p.101.

[8] T.K.Gaisser and T.Stanev, Nucl.Inst.Meth. A 235, 183, (1985).

[9] C.Forti et al., Phys.Rev. D 42, 3668, (1990).

[10] H.M.Hillas, in Proc.17th Int.Cosmic Ray Conf. (Paris) 8, 193, (1981).

[11] B.Rossi, Rev.Mod.Phys. 13, 240, (1941). 'High Energy Particles', Prentice Hall, Englewood Cliffs, NJ, (1952).

[12] P.Lipari, Astroparticle Physics 1, 195, (1993).

[13] V.I.Smirnov. "A course in higher mathematics" Pergamon Press, Oxford, (1964).

[14] Paolo Lipari \& Todor Stanev, Phys.Rev. D 44 (1991) 3543.

[15] J.A.Wrotniak, Showersim/84, University of Maryland report 85-191 (1984) unpublished. 